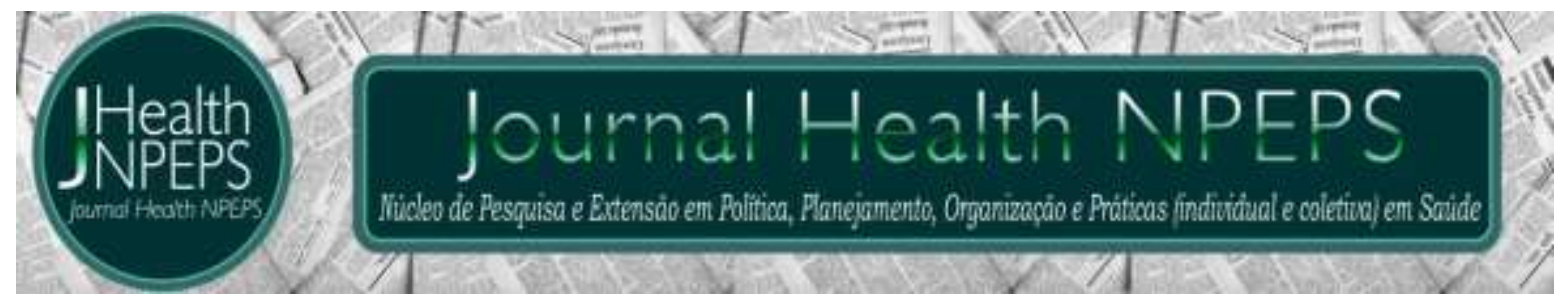

http://dx.doi.org/10.30681/252610103344

ARTIGO ORIGINAL

\title{
Perfil hídrico e consumo de suplementos por frequentadores de academias
}

\author{
Water profile and consumption of supplements by academics
}

Perfil hídrico y consumo de suplementos por frecuentadores de gimnasios

\begin{abstract}
Alan Talles Botelho ${ }^{1}$, Enio Pereira Soares ${ }^{2}$, Vanessa Santos Silva ${ }^{3}$, Letícia Josyane Ferreira Soares ${ }^{4}$, Luana Lemos Leão ${ }^{5}$, Priscila Avelar Monteiro ${ }^{6}$, Suzy Alice Souza ${ }^{7}$, Aline Fernandes de Souza ${ }^{8}$, Fernanda Gabriele Santos Rocha ${ }^{9}$, Paula Karoline Soares Farias ${ }^{10}$
\end{abstract}

\begin{abstract}
RESUMO
Objetivo: avaliar o perfil hídrico e o consumo de suplementos de pessoas fisicamente ativas inseridas em academias da cidade Montes Claros, Minas Gerais, Brasil. Método: Trata-se de estudo transversal, realizado com praticantes de atividade física, em 2018. Participaram da pesquisa 200 pessoas, maiores de 20 anos, de ambos os sexos, frequentadores de academias. Realizou-se a avaliação nutricional dos participantes, com aplicação de questionário estruturado, contendo questões relacionadas à prática de atividade física, ingestão hídrica e uso de suplementos alimentares. Resultados: dentre
\end{abstract}

\footnotetext{
${ }^{1}$ Nutricionista. Faculdades Unidas do Norte de Minas - FUNORTE. Montes Claros, MG, Brasil. E-mail: allan talles@hotmail.com ORCID ID: https://orcid.org/0000-0003-1814-2712

${ }^{2}$ Nutricionista. Faculdades Unidas do Norte de Minas - FUNORTE. Montes Claros, MG, Brasil. E-mail: eniopereira128@yahoo.com ORCID ID: https://orcid.org/0000-0002-4724-5844

${ }^{3}$ Nutricionista. Faculdade de Saúde Ibituruna - FASI. Montes Claros, MG, Brasil. E-mail: vanaurora@gmail.com ORCID ID: https://orcid.org/0000-0002-0520-5107

${ }^{4}$ Nutricionista. Mestre em Produção Animal. Universidade Federal de Minas Gerais - UFMG. Montes Claros, MG, Brasil. E-mail: leticiasoares.nutricionista@yahoo.com ORCID ID: https://orcid.org/0000-0002-2427-6453

${ }^{5}$ Nutricionista. Doutoranda em Ciências da Saúde. Universidade Estadual de Montes Claros - UNIMONTES. Montes Claros, MG, Brasil. E-mail: luanalemosleao@outlook.com ORCID ID: https://orcid.org/000-0003-3734-6964

${ }^{6}$ Nutricionista. Mestranda em Ciências da Saúde pela Universidade Estadual de Montes Claros - Unimontes. Montes Claros, MG, Brasil. E-mail: avelar monteiro@hotmail.com ORCID ID: https://orcid.org/0000-0001-6044-0954

${ }^{7}$ Nutricionista. Faculdade de Saúde Ibituruna - FASI. Montes Claros, MG, Brasil. E-mail: sasnutri@hotmail.com ORCID ID: https://orcid.org/0000-0002-6071-7535

${ }^{8}$ Farmacêutica. Mestranda em Ciências da Saúde pela Universidade Estadual de Montes Claros - UNIMONTES. Montes Claros, MG, Brasil. E-mail: alineffarma@gmail.com ORCID ID: https://orcid.org/0000-0001-7201-6042

${ }^{9}$ Assistente Social. Graduanda em Nutrição. Faculdade de Saúde Ibituruna - FASI. Montes Claros, MG, Brasil. E-mail: fernandagau@hotmail.com ORCID ID: https://orcid.org/0000-0002-4959-663X

${ }^{10}$ Nutricionista. Doutoranda em Ciências da Saúde. Universidade Estadual de Montes Claros - UNIMONTES. Montes Claros, MG, Brasil. E-mail: paulak.soares@hotmail.com ORCID ID: https://orcid.org/0000-0003-2967-5469 Autor principal - Endereço para correspondência: Secretaria de Educação de Montes Claros, Rua Francisco Coutinho, no 457, Augusta Mota, CEP: 39403219 - Montes Claros, MG, Brasil.
}

Journal Health NPEPS. 2019 jan-jun; 4(1):258-268. ISSN 2526-1010

Este artigo está licenciado sob forma de uma licença Creative Commons Atribuição 4.0 Internacional, que permite uso irrestrito, distribuição e reprodução em qualquer meio, desde que a publicação original seja corretamente citada. 
os participantes, $52,5 \%$ era do sexo feminino e $65 \%$ estavam com peso adequado. Verificou-se que a $71 \%$ da população estuda consumia mais que dois litros de água por dia. Dentre os praticantes de atividade física, $60,5 \%$ e $99 \%$ não faziam o uso de suplementos e anabolizantes, respectivamente. Além disso, $96 \%$ não utilizavam ergogênicos. Conclusão: este estudo demonstrou que a população estudada possui ingestão adequada de água e baixo consumo de suplementos, anabolizantes e ergogênicos.

Descritores: Academias de Ginástica; Exercício; Suplementos Nutricionais.

\section{ABSTRACT}

Objective: to evaluate the water profile and the consumption of supplements by physically active people inserted in academies of the city of Montes Claros, Minas Gerais, Brazil. Method: This is a cross-sectional study, carried out with physical activity practitioners in 2018. The study included 200 people, over 20 years old, of both sexes, attending academies. The nutritional evaluation of the participants was carried out with the application of a structured questionnaire containing questions related to the practice of physical activity, water intake and use of dietary supplements. Results: among the participants, 52.5\% were female and $47.5 \%$ male. Among all respondents, $65 \%$ had normal weight. It was found that $71 \%$ of the study population consumed more than two liters of water per day. Among practitioners of physical activity, $60.5 \%$ and 99\% were not using supplements and steroids, respectively. In addition, $96 \%$ of the participants did not use ergogenics. Conclusion: this study demonstrated that the population had adequate water intake and low intake of supplements, steroids and ergogenics.

Descriptors: Fitness Centers; Exercise; Food Supplements.

\section{RESUMEN}

Objetivo: evaluar el perfil hídrico y el consumo de suplementos de personas físicamente activas insertadas en gimnasios de la ciudad Montes Claros, Minas Gerais, Brasil. Método: Se trata de un estudio transversal, realizado con practicantes de actividad física, en 2018. Participaron de la investigación 200 personas, mayores de 20 años, de ambos sexos, frecuentadores de gimnasios. Se realizó la evaluación nutricional de los participantes, con aplicación de cuestionario estructurado, conteniendo cuestiones relacionadas a la práctica de actividad física, ingestión hídrica y uso de suplementos alimenticios. Resultados: de los participantes, el 52,5\% era del sexo femenino y el 47,5\% del sexo masculino. Entre todos los entrevistados, el 65\% estaba con peso adecuado. Se verificó que el $71 \%$ de la población estudia consumía más de dos litros de agua al día. Entre los practicantes de actividad física, el 60,5\% y el 99\% no hacían el uso de suplementos y anabolizantes, respectivamente. Además, el $96 \%$ de los estudiados no utilizaba ergogénicos. Conclusión: este estudio demostró que la población estudiada posee ingestión adecuada de agua y bajo consumo de suplementos, anabolizantes y ergogénicos.

Descriptores: Gimnasios; Ejercicio; Suplementos Nutricionales.

\section{INTRODUÇÃO}

A busca por uma vida saudável, com alimentação balanceada associada à prática de exercícios físicos vem crescendo tanto entre aqueles que antes só se preocupavam com a estética, 
quanto nos outros grupos com maior preocupação em relação ao bem-estar ${ }^{1}$. A popularidade dos suplementos alimentares aumentou bastante nos últimos anos. Com isso, desenvolveu-se uma cultura sobre esses suplementos não apenas entre os esportistas e profissionais, mas também entre as pessoas fisicamente ativas ${ }^{2}$.

As substâncias mais comumente utilizadas são os suplementos alimentares, no qual apresentam uma ênfase primordial na definição corporal ${ }^{3}$. Um excelente desempenho na prática de exercício físico com a utilização de pesos, pode ser obtido adotando-se uma alimentação adequada quanto à qualidade, quantidade e horário corretos da ingestão, aliada a reposição hidroeletrolítica antes e após o treino ${ }^{4}$.

Os suplementos alimentares são meios complementares que podem ser utilizados para a melhoria no desempenho das atividades esportivas, visando reduzir a fadiga causada pelo estímulo do músculo. São compostos por vitaminas, minerais, aminoácidos, proteínas, carboidratos, que atuam na prevenção de carências nutricionais ${ }^{5}$. As pessoas fisicamente ativas tendem a obter informações de mídias eletrônicas ou através de amigos, ao invés de consultar fontes seguras e profissionais da área. E com base em informações superficiais e/ou incertas, essas pessoas utilizam esses suplementos com percepções de benefícios irreais sobre seus impacto ${ }^{6}$.

Outro fator importante para frequentadores de academias é a hidratação, reposição de água. Esta, deve ocorrer a cada 15 minutos de exercícios físicos, durante todo o treino. A ingesta de água supre células de substâncias energéticas e construtoras, auxilia na eliminação de resíduos metabólicos, mantém o equilíbrio ácidobásico e regulam a temperatura corporal ${ }^{7}$.

O exercício físico leva a um acréscimo significativo na temperatura do corpo interna e externa, o que gera o aumento da excreção e circulação de suor, que é composto por água e eletrólitos. Caso não ocorra a reposição de líquidos e eletrólitos após o exercício físico, o organismo terá risco maior de perda de força, câimbras e amplo prejuízo no desempenho ${ }^{8}$. A prática de exercício tem sido um grande aliado para os fatores de prevenção, reversão e controle de diversas patologias principalmente as doenças crônicas não transmissíveis. E o ambiente da academia caracteriza-se por essa busca na manutenção e promoção à saúde?. 
Diante disso, o presente estudo objetivou avaliar avaliar o perfil hídrico e o consumo de suplementos de pessoas fisicamente ativas inseridas nas academias da cidade Montes Claros (MG), Brasil.

\section{MÉTODO}

Trata-se de estudo transversal, realizado com praticantes de atividade física, de ambos os sexos, de academias particulares no município de Montes Claros (MG), durante o ano de 2018.

A amostra foi composta por 200 praticantes de atividade física, que concordaram em participar da pesquisa e assinaram o Termo de Consentimento Livre e Esclarecido (TCLE). Antes da coleta de dados, foi solicitada a autorização das academias participantes.

Foi realizado um sorteio das academias registradas no município, e destas foram sorteadas, de maneira aleatória simples, cinco academias. Os critérios de inclusão foram: estar devidamente matriculado(a) e frequente na academia e ter idade superior ou igual a 20 anos.

Os de exclusão foram: não ser o português o idioma nativo do participante, problemas de visão e/ou de audição (relatados/percebidos), intoxicação por álcool e/ou outras drogas no momento da entrevista.

Os dados antropométricos de peso e estatura dos praticantes de atividade física foram coletados de acordo com o preconizado pelo Sistema de Vigilância Alimentar e Nutricional $(\text { SISVAN })^{10}$. 0 peso foi obtido através de uma balança portátil (Plenna®) e a altura foi mensurada através de um estadiômetro portátil (Sanny®).

Para diagnóstico do estado nutricional segundo o IMC, foi utilizada a recomendação para adultos ( $\geq 20$ anos e $<60$ ), proposto pela Organização Mundial da Saúde $(\mathrm{OMS})^{11}$. Esta recomendação classifica os pontos de corte de IMC menor que 18,5 como desnutrição; de 18,5 a 24,9 como eutrofia; de 25,0 a 29,9 como sobrepeso e acima de 30,0 como obesidade.

0 consumo de água, suplementos, ergogênicos e anabolizantes pelos frequentadores da academia foram avaliados utilizando o questionário baseado em autores de referência ${ }^{11}$, com alterações, nos quais foram observadas questões específicas adaptadas para o estudo.

Para efeito de análise, os praticantes de atividade física foram divididos de acordo com gênero e a idade. Para análise estatística descritiva, 
utilizou-se o programa Microsoft Excel $®$ de 2007 em combinação com o software BioEstat $\circledR^{2}$ versão 5.3, apresentando os dados em tabelas, com números absolutos e relativos.

Este estudo foi aprovado pelo Comitê de Ética e Pesquisa em Seres Humanos com o número 1.718.549, e está em acordo com a Resolução 466/12 do Conselho Nacional de Saúde, que regulamenta as normas aplicadas às pesquisas que envolvem seres humanos.

\section{RESULTADOS}

Foram avaliados 200 praticantes de atividade física, sendo desses, 52,5\% do sexo masculino $(n=105)$ e $47,5 \%$ do sexo feminino $(n=95)$. Em relação ao IMC, observou-se que 65\% ( $n=130)$ dos praticantes de atividade física se encontravam em peso adequado $e$ dentre esses, a maioria $(33 \%$ / $\mathrm{n}=66)$ eram mulheres (Tabela 1 ).

Tabela 1- Índice de massa corporal em relação o sexo. 2018. Montes Claros-MG, Brasil.

\begin{tabular}{lcccccc}
\hline IMC & \multicolumn{2}{c}{ Masculino } & \multicolumn{2}{c}{ Feminino } & Total \\
& $\mathrm{n}$ & $\%$ & $\mathrm{n}$ & $\%$ & $\mathrm{~N}$ & $\%$ \\
Baixo Peso & 5 & 2,5 & 5 & 2,5 & 10 & 5 \\
Eutrofia & 64 & 32 & 66 & 33 & 130 & 65 \\
Sobrepeso & 24 & 12 & 17 & 8,5 & 41 & 20,5 \\
Obesidade & 12 & 6 & 7 & 3,5 & 19 & 9,5 \\
Total & 105 & 52,5 & 95 & 47,5 & 200 & 100 \\
\hline
\end{tabular}

As informações referentes ingestão hídrica, suplementos alimentares, anabolizantes e ergogênicos encontram-se na Tabela 2.

Tabela 2- Avaliação do consumo de água, suplementos, anabolizantes e ergogênicos. 2018. Montes Claros-MG, Brasil.

\begin{tabular}{|c|c|c|}
\hline Variáveis & $\mathbf{n}$ & $\%$ \\
\hline \multicolumn{3}{|c|}{ Consumo de água } \\
\hline $\begin{array}{l}<\text { dois } \\
\text { litros/dia }\end{array}$ & 58 & 29 \\
\hline $\begin{array}{l}\text { > dois } \\
\text { litros/dia }\end{array}$ & 142 & 71 \\
\hline \multicolumn{3}{|c|}{ Suplementos } \\
\hline Sim & 79 & 39,5 \\
\hline Não & 121 & 60,5 \\
\hline \multicolumn{3}{|c|}{ Anabolizantes } \\
\hline Sim & 2 & 1 \\
\hline Não & 198 & 99 \\
\hline \multicolumn{3}{|c|}{ Ergogênicos } \\
\hline Sim & 8 & 4 \\
\hline Não & 192 & 96 \\
\hline
\end{tabular}

Os suplementos alimentares mais consumidos foram à base de proteína, carboidratos, além dos aminoácidos ramificados (BCAA). 0 consumo de shakes para substituir refeições também foi relatado por parte dos entrevistados. Após a afirmação do consumo do suplemento, foram avaliados quais seriam esses suplementos, e observou-se a ingestão de mais de um suplemento alimentar relatado pelos participantes (Tabela 3).

Outro fator observado foi que $83,5 \%(n=66)$ dos participantes faziam o uso dos suplementos alimentares por iniciativa própria e 16,5\% (n=13) por indicação de um amigo(a). 
Tabela 3 - Consumo de suplementos alimentares por sexo. 2018. Montes Claros-MG, Brasil.

\begin{tabular}{|c|c|c|c|c|}
\hline \multirow[t]{2}{*}{$\begin{array}{l}\text { Suplemento } \\
\text { alimentar }\end{array}$} & \multicolumn{2}{|c|}{$\begin{array}{l}\text { Masculino } \\
(n=49)\end{array}$} & \multicolumn{2}{|c|}{$\begin{array}{c}\text { Feminino } \\
(n=30)\end{array}$} \\
\hline & $\mathrm{n}$ & $\%$ & $\mathrm{n}$ & $\%$ \\
\hline $\begin{array}{l}\text { Ricos em } \\
\text { Proteínas }\end{array}$ & 15 & 30.6 & 12 & 40,0 \\
\hline Glutamina & 8 & 16,3 & 3 & 10,0 \\
\hline $\begin{array}{l}\text { Shakes para } \\
\text { substituir } \\
\text { refeições }\end{array}$ & 5 & 10,2 & 22 & 73,3 \\
\hline $\begin{array}{l}\text { Hipercalóricos ou } \\
\text { Compensadores }\end{array}$ & 7 & 14,3 & 3 & 10,0 \\
\hline Creatina & 3 & 6,1 & 4 & 13,3 \\
\hline Bebidas Isotônicas & 35 & 71,4 & 11 & 36,7 \\
\hline $\begin{array}{l}\text { Ricos em } \\
\text { Carboidratos }\end{array}$ & 21 & 42,8 & 8 & 26,7 \\
\hline $\begin{array}{l}\text { Naturais e } \\
\text { Fitoterápicos }\end{array}$ & 4 & 8,1 & 16 & 56,3 \\
\hline $\begin{array}{l}\text { Beta-Hidroxi } \\
\text { Beta-Metilbutirato } \\
\text { (HMB) }\end{array}$ & 6 & 12,2 & 2 & 6,7 \\
\hline $\begin{array}{l}\text { Vitaminas e } \\
\text { Minerais }\end{array}$ & 24 & 48,9 & 19 & 63,3 \\
\hline $\begin{array}{l}\text { Ácido Linoleico } \\
\text { Conjugado (CLA) }\end{array}$ & 6 & 12,2 & 1 & 3,3 \\
\hline $\begin{array}{l}\text { Queimadores de } \\
\text { gorduras }\end{array}$ & 8 & 16,3 & 17 & 56,7 \\
\hline $\begin{array}{l}\text { Aminoácidos } \\
\text { ramificados } \\
\text { (BCAA) }\end{array}$ & 22 & 44,9 & 3 & 10,0 \\
\hline $\begin{array}{l}\text { Outros } \\
\text { aminoácidos } \\
\text { (líquido ou em } \\
\text { cápsula) }\end{array}$ & 13 & 26,5 & 3 & 10,0 \\
\hline
\end{tabular}

\section{DISCUSSÃO}

No presente estudo, pode-se verificar que a maioria dos praticantes de atividade física era do sexo masculino. No entanto, Oliveira et $\mathrm{al}^{13}$ verificaram que a maioria dos frequentadores da academia são do sexo feminino, assim como o estudo de Adam et $\mathrm{al}^{14}$ o qual aponta $62,9 \%$ como sendo desse público.

Journal Health NPEPS. 2019 jan-jun; 4(1):258-268.
A antropometria é um dos procedimentos de maior aplicabilidade para avaliação nutricional, devido seu baixo custo, simplicidade de utilização e aceitabilidade do método ${ }^{15}$. De acordo com achados do estudo, a maioria da população estudada, tanto homens quanto mulheres apresentaram peso adequado de acordo com o critério de classificação do IMC. Estudo realizado em São Bento do Uma (PE), observou que $60 \%$ da população encontrava-se com o peso adequado ${ }^{1}$. Outros pesquisadores ${ }^{15}$ reforçam esses achados, ao revelar $44 \%$ em estado de eutrofia.

A ingestão hídrica diária da maioria dos participantes foi maior de dois litros. Corroborando com isso, alguns pesquisadores ${ }^{15,17}$ confirmam este perfil de consumo de água em outras regiões do Brasil. É de conhecimento que a reposição hídrica é uma necessidade que está diretamente relacionada com a intensidade, duração e a temperatura do local da prática do exercício ${ }^{16}$. Assim, a reposição de líquidos apropriada é recomendada como primeiro passo na prevenção de futuros danos ao organismo durante as atividades físicas $^{18}$.

Existem algumas divergências em relação à quantidade de água a ser ingerida diariamente por praticantes de 
atividade física. Pesquisadores ${ }^{3}$ defendem que essa ingestão deve ser feita de acordo com a sede do indivíduo, uma vez que Sistema Nervoso Central (SNC) pode ser capaz de indicar corretamente o volume de líquido a ser ingerido. Entretanto, de acordo com Ferreira et $\mathrm{al}^{19}$ a melhor forma de hidratação é a ingestão de $3 \mathrm{ml} / \mathrm{kg}$ de peso corporal, assim o indivíduo poderá atingir os níveis máximos de sudorese (1 a $2 \%$ do peso corporal), sem apresentar desconforto gastrointestinal.

Em relação à ingestão de suplementos, observou-se que $39,5 \%$ faziam 0 uso. Todavia, outros estudos ${ }^{1,13,15}$ apontam consumo mais alto de suplementos. Dessa forma, evidenciase que esses frequentadores de academia se expõem a alguns riscos, visto que muitos substituem os alimentos convencionais da dieta por estes produtos $^{20}$.

Assim como os suplementos, os ergogênicos também são frequentemente utilizados por praticantes de atividades físicas ${ }^{21}$. No presente estudo, verificou-se que apenas $4 \%$ dos entrevistados faziam uso. Esse achado é distinto de estudo anterior, no qual $73 \%$ dos entrevistados consumiam algum tipo de suplemento com 0 objetivo de obter os efeitos dos ergogênicos ${ }^{22}$.

Dentre os participantes do presente estudo, apenas 1\% afirmou fazer uso de anabolizantes. Dado similar foi encontrado por Dal Pizzol et $\mathrm{al}^{23} \mathrm{em}$ Passo Fundo (RS), em que constataram que $2,2 \%$ dos entrevistados declararam já ter usado. Mineiro et al ${ }^{24}$ ao avaliaram o consumo de anabolizantes, verificam que $21,95 \%$.

No Brasil, entre os anos de 2000 a 2010, ocorreram 1.319 internações por ingestão de anabolizantes; $1 \%$ dos pacientes evoluiu para óbito ${ }^{25}$. Segundo Tavares et $\mathrm{al}^{26}, \quad \mathrm{o}$ consumidor preferencial de anabolizante no Brasil são os homens, com idade entre 18 e 34 anos, cuja administração das drogas ocorre em quantidades superiores às terapêuticas e com associação de diferentes tipos.

Neste contexto, o uso de suplementos alimentares aumentou largamente e os motivos para esse crescimento, apoia-se no marketing do corpo perfeito, logo a busca por uma imagem que segue padrões sociais e comerciais. Particularmente, os homens e os atletas, constituem o maior grupo consumidor desses produtos. $E$ entre os frequentadores de academias, a justificativa para o uso desses 
suplementos relaciona a melhora da resposta muscular ao treinamento de resistência ${ }^{27}$.

\section{CONCLUSÃO}

Verifica-se a prevalência do sexo masculino na pesquisa e no consumo dos suplementos alimentares. A maioria dos participantes apresentam eutrofia e consumiam mais de dois litros de água por dia. Entretanto, outros estudos são necessários para se avaliar os hábitos alimentares dessa população, associado com o consumo de suplementos alimentares.

Além disso, deve-se atentar para a importância do nutricionista na academia, visto que este profissional é especializado para desenvolver estratégias de orientações nutricionais e adequar o consumo de nutrientes de forma individual, com o objetivo de contribuir para o melhor desempenho da pessoa fisicamente ativa.

\section{REFERENCIAS}

1. Costa WS. A avaliação do estado nutricional e hábitos alimentares de alunos praticantes de atividade física de uma academia d município de São
Bento do Una - PE. Rev Bras Nutr Esp. 2012; 6(36):464-469.

2. Goston JL, Correia MI. Intake of nutritional supplements among people exercising in gyms and influencing factors. Nutr. 2010; 26(6): 604-611.

3. Moreira MAC, Gomes VCA, Garcia SE, Rodrigues COL. Hidratação durante o exercício: a sede é suficiente? Rev Bras Med Esporte. 2006; 12(6):405409.

4. Souza JÁ, Navarro F. Avaliação do perfil antropométrico e nutricional de atletas de futsal do clube Rio BrancoES. Rev Bras Nutr Esp. 2015; 9(50):111-119.

5. Lopes IR, Souza TPM, Quintão DF. Uso de suplementos alimentares e estratégias de perda ponderal em atletas de jiu-jitsu de Ipatinga-MG. Rev Bras Nutr Esp. 2014; 8(46):254263.

6. Hartmann C, Siegrist M. Benefit beliefs about protein supplements: $A$ comparative study of users and nonusers. Appetite. 2016; 103:229-235.

7. Gracinao LC, Ferreira FG, Chapeta SMSV, Scolforo LB, Segheto W. Nível de conhecimento e prática de hidratação em praticantes de atividade física em academia. Rev Bras Nutr Esp. 2014; 8(45):146-155. 
8. Loiola PC, Benetti MV, Durante JG, Frade RE, Viebig RF. Avaliação da porcentagem de perda de peso e taxa de sudorese após o treino de lutadores de uma academia no estado de São Paulo. Rev Bras Nutr Esp. 2015; 9(49):74-83.

9. Frade RET, Viebig RF, Moreira ICLS, Fonseca DC. Avaliação do consumo de suplementos nutricionais por frequentadores de uma academia da cidade de São Paulo-SP. Rev Bras Nutr Esp. 2016; 10(55):50-58.

10. Ministério da Saúde (BR). Vigilância alimentar e nutricional - SISVAN: Orientações para a coleta de dados antropométricos em serviços de Saúde. Brasília: Ministério da Saúde; 2011.

11. World Health Organization. Physical status: the use and interpretation of anthropometry. Geneva: WHO Technical Report Series; 1995.

12. Goston JL. Prevalência do uso de suplementos nutricionais entre praticantes de atividade física em academias de belo horizonte: fatores associados [dissertação]. Beo horizonte: Universidade Federal de Minas Gerais; 2008. 74 p.

13. Oliveira CE, Sandoval TC, Silva JCS, Stulbach TE, Frade RET. Avaliação do consumo alimentar antes da prática de atividade física de frequentadores de uma academia no município de São Paulo em diferentes modalidades. Rev Bras Nutr Esp. 2013; 7(37):57-67.

14. Adam BO, Fanelli C, Souza ES, Stulbach TE, Monomi PY. Conhecimento nutricional de praticantes de musculação de uma academia da cidade de São Paulo. Rev Bras Nutr Esp. 2013; 2(2):24-36.

15. Uchoas GDS, Pires CR, Marin T. Hábitos alimentares de frequentadores de academias em Apucarana-PR. Rev Bras Nutr Esp. 2011; 5(30):530-540.

16. Brito IP. Considerações atuais sobre reposição hidroeletrolítica no esporte. Nutr Pauta. 2003; 62:48-52.

17. Peres N, Reis GC, Silva CC, Viebig RF, Mendonça RB. Interesse e conhecimentos básicos em nutrição dos praticantes de atividade física de uma academia da região norte do município de São Paulo. Rev Dig. 2009; 14(134):1-12.

18. Furtado MC, Garcia MJ, Gonçalves PJ, Viebig FR. Avaliação de hábitos e conhecimentos sobre hidratação de praticantes de musculação uma academia da cidade de São Paulo. Rev Dig. 2009; 14(133):1-10. 
19. Ferreira FG, Alves $K$, Costa NMB, Santana AMC, Marins JCB. Efeito do nível de condicionamento físico e da hidratação oral sobre a homeostase hídrica em exercício aeróbico. Rev Bras Med Esporte. 2010; 16(3):166170.

20. Araújo LR, Andreolo J, Silva MS. Utilização de suplemento alimentar e anabolizante por praticantes de musculação nas academias de Goiânia-GO. Rev bras ciênc mov. 2002; 10(3):13-18.

21. Neto TLB. A Controvérsia dos Agentes Ergogênicos: Estamos Subestimando os Efeitos Naturais da Atividade Física? Arq bras endocrinol metab. 2001; 45(2):121-122.

22. Freitas A, Evangelista AL, Lopes CR, Silva AKS, Lima AV, Freitas ESF. Uso de suplementos ergogênicos em praticantes de atividades esportivas na cidade de Teresina-PI. Rev Bras Nutr Esp. 2013; 7(40):246-252.

23. Dal Pizzol TDS, Branco MMN, Carvalho RMAD, Pasqualotti A, Maciel EN, Migott AMB. Non-medical use of psychoactive medicines among elementary and high school students in Southern Brazil. Cad Saúde Pública. 2006; 22(1):109-115.

24. Mineiro L, Silva JC, Machado Júnior $\mathrm{O}$, Rocha RE. Uso de esteroides por frequentadores de academias de musculação na cidade de CaçadorSC. Rev bras prescrição fisiol exercício. 2015; 9(53):321-327

25. Silva Junior SHA. Morbidade hospitalar por ingestão de esteroides anabólico-androgênicos (EAA) no Brasil. Rev Bras Med Esporte. 2013; 19(2):108-111.

26. Tavares FAG, Suffredini TS, Oliveira CCES, Biagini AP, Oliveira NML. Atuação dos esteróides anabolizantes na regeneração musculoesquelética. Arq Ciências Saúde UNIPAR. 2008; 15(3):145-149.

28. Saidi O, Ayed IB-B, Benzarti A, Duchéa $P$, Serairi $R$. Intake of carbohydrate-protein supplements by recreational users at gyms: Body composition improved?. Sci sports. 2018; 33:141-149. 
Botelho AT, Soares EP, Silva VS, Soares LJF, Leão LL, Monteiro PA, et al. Perfil hídrico e consumo de suplementos...

Conflito de interesses: Os autores declaram não haver conflito de interesses.

\section{Participação dos autores:}

- Concepção: Botelho AT, Soares EP, Farias PKS.

- Desenvolvimento: Botelho AT, Soares EP, Silva VS, Soares LJF, Leão LL, Monteiro PA, Souza SA, Souza AF, Rocha FGS, Farias PKS.

- Redação e revisão: Botelho AT, Soares EP, Silva VS, Soares LJF, Leão LL, Monteiro PA, Souza SA, Souza AF, Rocha FGS, Farias PKS.

Como citar este artigo: Botelho AT, Soares EP, Silva VS, Soares LJF, Leão LL, Monteiro PA, et al. Perfil hídrico e consumo de suplementos por frequentadores de academias. J Health NPEPS. 2019; 4(1):258-268.

Submissão: $30 / 01 / 2019$

Aceito: $15 / 05 / 2019$

Publicado: 01/06/2019 